Sandra Lynn Jaya-Bodestyne*, Lai Heng Lee, Lay Kok Tan, Kok Hian Tan, Truls Østbye, Rahul Malhotra, John Allen, Sophie Seine Xuan Tan, Melinda Si Yun Tan, Lawrence Cheng Kiat Ng, Yang Yong and Thiam Chye Tan

\title{
Risk factors for pregnancy-associated venous thromboembolism in Singapore
}

https://doi.org/10.1515/jpm-2020-0298

Received April 10, 2020; accepted August 10, 2020; published online September 5, 2020

\begin{abstract}
Objectives: Pregnancy-associated venous thromboembolism (VTE), including deep venous thrombosis (DVT) and pulmonary embolism (PE), is associated with increased risk of maternal mortality and morbidity. This study aimed to assess potential risk factors for pregnancy-associated VTE.
\end{abstract}

Methods: In this case-control study, women with pregnancy-associated VTE were identified via International Classification of Diseases codes and included if they had been objectively diagnosed with VTE during pregnancy or within six weeks postpartum, from 2004 to 2016, at KK Women's and Children's Hospital or Singapore General Hospital in Singapore. Controls, i.e. pregnant women without VTE, were selected from a prospective longitudinal study. The odds ratio (OR) for VTE was computed for a range of maternal and obstetric factors.

Results and conclusions: From 2004 to 2016, 89 cases of pregnancy-associated VTE and 926 controls were identifed and analysed using logistic regression. The most significant risk factors for pregnancy-associated VTE were

*Corresponding author: Sandra Lynn Jaya-Bodestyne, Duke-NUS Medical School, 8 College Road, Singapore 169857, Singapore. Phone: +6593669155, E-mail: sandra.jayabodestyne@mohh.com.sg Lai Heng Lee, Melinda Si Yun Tan and Lawrence Cheng Kiat Ng, Department of Haematology, Singapore General Hospital, Singapore, Singapore

Lay Kok Tan, Department of Obstetrics and Gynaecology, Singapore General Hospital, Singapore, Singapore

Kok Hian Tan and Thiam Chye Tan, Department of Obstetrics and Gynaecology, KK Women's and Children's Hospital, Singapore, Singapore

Truls Østbye, Rahul Malhotra and John Allen, Duke-NUS Medical School, Singapore, Singapore

Sophie Seine Xuan Tan, Yong Loo Lin School of Medicine, National University of Singapore, Singapore, Singapore

Yang Yong, Department of Epidemiology, Singapore General Hospital, Singapore, Singapore smoking (OR 5.44, $\mathrm{p}=0.0002$ ) and preterm delivery (OR $5.06, \mathrm{p}=0.023)$. Malay race, multiparity, non-O blood group and caesarean section, were also identified to be of higher risk. These risk factors should be useful in the development of thromboprophylaxis strategies for pregnancy and the postpartum period, especially in Singapore.

Keywords: postpartum; pregnancy; risk factors; venous thromboembolism.

\section{Introduction}

\section{Pregnancy-associated venous thromboembolism}

Venous thromboembolism (VTE), which includes deep vein thrombosis (DVT) and pulmonary embolism (PE), is 4-6 times more common in pregnant women compared to age-matched non-pregnant women $[1,2]$. This is attributed to the physiological state of pregnancy that exhibits all of the components of Virchow's triad of hypercoagulability, venous stasis, and vascular damage [3].

Although pregnancy-associated VTE occurs infrequently $(1.2-1.4$ per 1,000 deliveries $[4,5])$, it is one of the leading causes of maternal deaths in developed countries [6] and was the second most common cause of maternal deaths in Singapore from 1990 to 1999 [7]. Apart from mortality, pregnancy-associated VTE is also associated with long-term morbidity from post-thrombotic syndrome [8] and poorer quality of life [9].

\section{Risk factors for pregnancy-associated VTE}

Current recommendations for thromboprophylaxis during pregnancy and the postpartum period were adopted from the Royal College of Obstetricians and Gynecologists (RCOG) Green-top Guidelines [10], which are based on data from predominantly Western populations. Although widely accepted and employed across Asia [11], there are limitations in its utility. These include inconsistencies in 
the published literature with regards to the risk factors for pregnancy-associated VTE, e.g., while thrombophilia and previous VTE are two well-recognised significant risk factors for pregnancy-associated VTE, the evidence relating to factors such as age [12], parity [13] and diabetes [14] are conflicting. Variations in the strength of pregnancyassociated VTE risk factors such as obesity and caesarean section have also been reported [14]. The current risk assessment model does not include other factors which have been shown to be associated with an increased risk of pregnancy-associated VTE, such as non-O blood group [15] and newborn low birth weight (LBW) [16]. Lastly, the paucity of data on pregnancy-associated VTE and lack of validation in the racially diverse Singaporean population limits the applicability of the current risk assessment model.

The aim of this study was to assess potential risk factors for pregnancy-associated VTE in the obstetric population in Singapore, using a case-control approach.

\section{Materials and methods}

\author{
Study design, case definition and data collection
}

This case-control study includes data spanning 13 years, from 2004 to 2016, from two tertiary maternity hospitals (KK Women's and Children's Hospital (KKH) and Singapore General Hospital (SGH)) in Singapore Health Services, the largest healthcare group in Singapore. The study was approved by the Ethics Committee SingHealth Centralised Institutional Review Board (CIRB) on 26 August 2016 (reference number 2016/2343).

The outcome was defined as a first occurrence of VTE during pregnancy or the postpartum period (six weeks after delivery). The women with VTE (the "cases") were identified via International Classification of Diseases (ICD) codes, and included if they were objectively diagnosed via duplex ultrasound for DVT and computed tomography pulmonary angiography (CTPA), ventilation-perfusion (V/Q) scan, or autopsy for PE. Women with known high-risk thrombophilia were excluded as thromboprophylaxis would have been recommended from the first trimester until six weeks postpartum, and they would have been monitored closely for the development of VTE. The "controls" consisted of women from the Neonatal and Obstetric Risk Assessment (NORA) cohort, which was a prospective longitudinal study providing data on women with no VTE who delivered in KKH from September 2010 to August 2014 [17].

Medical records were reviewed, and information on 12 potential maternal and obstetric risk factors related to the pregnancy-associated VTE event was extracted. Maternal characteristics included race (Chinese, Malay, Indian, Others), age, body mass index (BMI), parity (number of births after 24 weeks gestation), blood group (O, A, B, AB), and smoking status (dichotomised as non-smoker or active smoker during pregnancy). Data on race was obtained from the identification document provided upon registration (i.e. National Registration Identity Card for Singaporean citizens and foreign citizens who are permanent residents of Singapore). As Chinese, Malay and Indian are the main racial groups in Singapore, other women were classified as 'Others'. BMI was calculated using the first recorded weight at an antepartum visit. The BMI classification for pre-pregnant populations [18] was used to determine the cut-off points for overweight and obesity. Obstetric characteristics included pre-eclampsia, diabetes mellitus (either pre-existing or gestational), mode of delivery (vaginal delivery, including forceps delivery and vacuum extraction, or caesarean section), gestational age at delivery, postpartum haemorrhage (defined as $\geq 500 \mathrm{~mL}$ ), and newborn LBW (defined as $<2500 \mathrm{~g}$ ).

\section{Statistical analysis}

Odds ratios (ORs) with 95\% confidence intervals (CIs) were calculated using logistic regression and tested for significance using a Wald $\chi^{2}$ test; p-values comparing proportions were calculated using Fisher's exact test. As the distribution of BMI was right-skewed and positive, the normalising transformation $\ln$ (BMI) was used in analysing differences between cases and controls. Due to departures from normality, group comparisons of gestational age at delivery and newborn birth weight were analysed using the Wilcoxon rank-sum test. Risk factors with a p-value of $<0.2$ in simple logistic regression analysis were included in a multivariable analysis with statistical significance set at $\mathrm{p} \leq 0.05$. All analyses were performed using SAS University Edition (SAS ${ }^{\circledR}$ Inc. Cary, NC USA).

\section{Results}

Eighty-nine pregnancy-associated VTE cases and 926 nonVTE controls were identified according to the inclusion and exclusion criteria (Figure 1). Of the 89 VTE cases, 18 were PE and 71 were DVT ( 4 were concurrent PE and DVT). 60 VTE cases occurred during the antepartum period, and 29 occurred during the postpartum period (Figure 2). A higher proportion of women with pregnancy-associated VTE were Malays, had parity of three or more, had non-O blood group, and were smokers (Table 1). The average age, BMI, proportion with pre-eclampsia, and proportion with diabetes mellitus were not statistically different for the cases and controls. Women with postpartum VTE were more likely to have a caesarean section, earlier delivery, postpartum haemorrhage and lower newborn birthweight compared to the controls.

Non-O blood group and smoking were statistically significant risk factors for pregnancy-associated VTE in the multivariable analysis (Table 2). Omnibus tests on race and parity did not achieve significance, however individual degree of freedom tests on Malay race and parity $\geq 3$ were significant and hence indicated as potential risk factors. We found no evidence for advanced maternal age, obesity, pre-eclampsia, and diabetes mellitus as risk factors of pregnancy-associated VTE. Caesarean section and preterm delivery were statistically significant risk factors for 


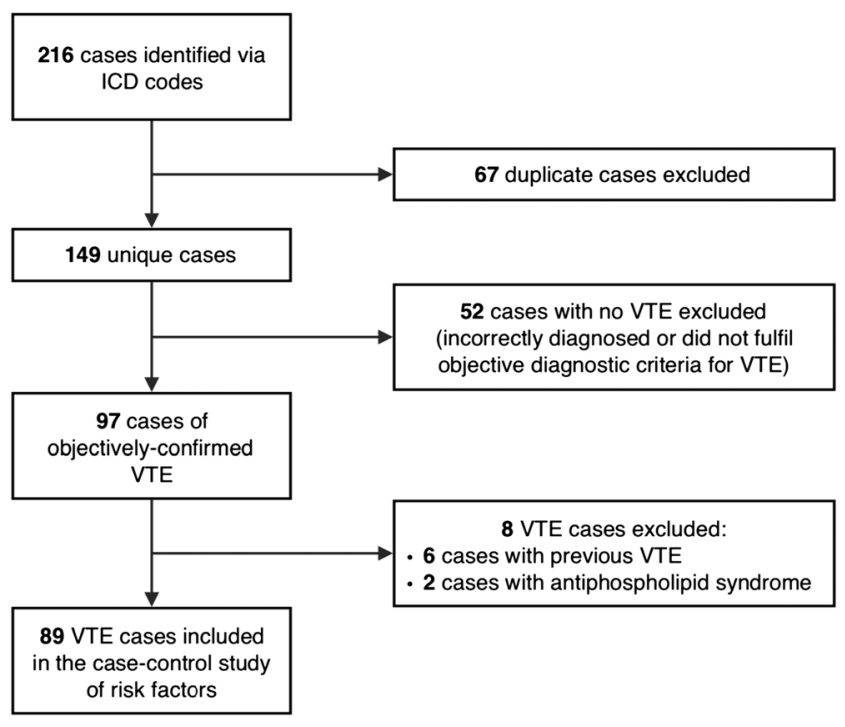

Figure 1: Flow chart of the study population. risk factors. The association between race and pregnancyassociated VTE has been studied extensively in Western countries, comparing African American, European, Hispanic and Asian subpopulations [19]. However, few studies have investigated variation in VTE risk among the different Asian subgroups. This is especially important in Singapore's heterogenous Asian population, which consists of three major racially and culturally distinct populations Chinese, Malay, and Indian. The increased VTE risk observed in Malay women compared to Chinese women supports local findings [19]. The reason for this difference in risk is likely multifactorial, with interactions between genetic factors such as undiagnosed thrombophilia, and environmental factors [20]. Non-O blood groups are recognised risk factors for thrombosis due to the higher levels of von Willebrand factor (VWF) and Factor VIII compared to those with $O$ blood group [21]. Advanced maternal age, obesity, and pre-eclampsia, which are commonly accepted

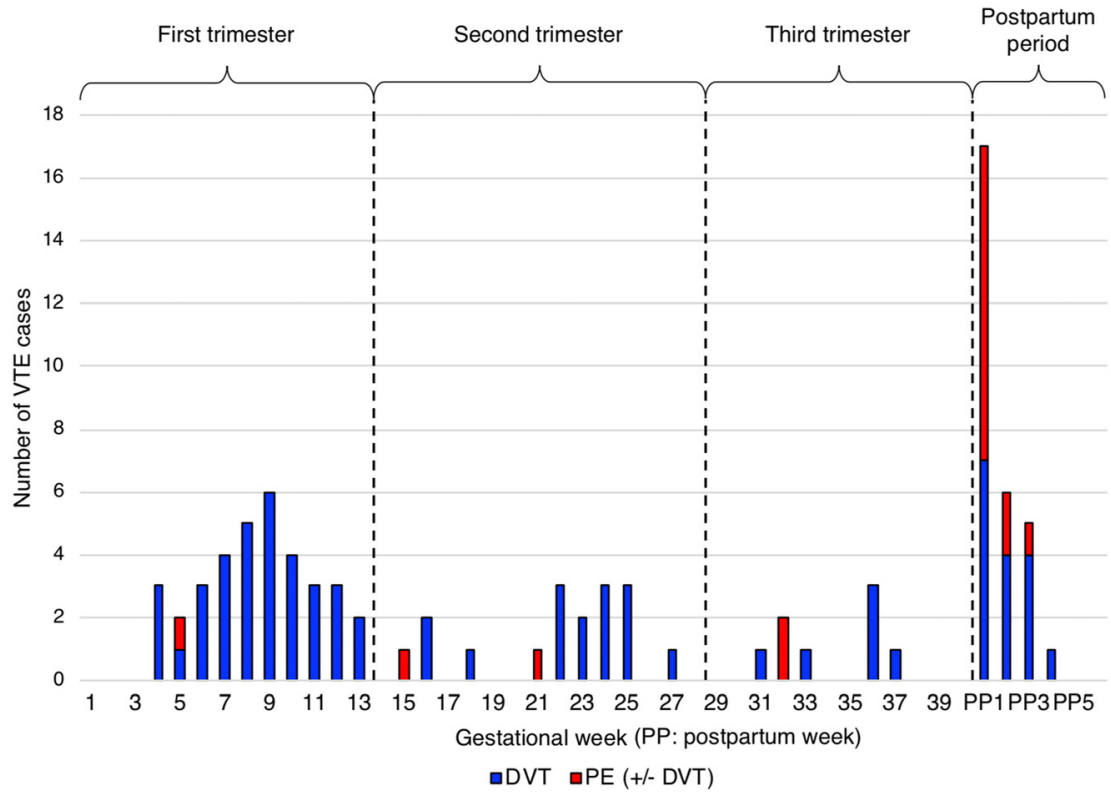

Figure 2: Distribution of pregnancyassociated VTE by week in pregnancy and postpartum. postpartum VTE. Postpartum haemorrhage, and low newborn birth weight were not found to be statistically significant risk factors for postpartum VTE.

\section{Discussion}

\section{Risk factors for pregnancy-associated VTE}

In this study, non-O blood group and smoking were statistically significant risk factors for pregnancy-associated VTE. Being Malay and parity $\geq 3$ were identified as potential risk factors for VTE, were interestingly not statistically associated with VTE risk in this study. This could reflect the relatively small sample size and thus these nonetheless continue to warrant consideration as clinically relevant risk factors. The static measurement of BMI could also account for its finding as a non-significant risk factor. Weight gain during pregnancy, which was not studied, could have potentially contributed to a woman's VTE risk. The role of diabetes mellitus in predisposing to VTE has been conflicting [22]. Although it was not found to be a risk factor in this study, it is possible that the risk of VTE be due to the metabolic syndrome, such as hypertensive disorders and 
Table 1: Subject characteristics (cases vs. controls).

\begin{tabular}{|c|c|c|c|}
\hline $\begin{array}{l}\text { Subject } \\
\text { characteristics }\end{array}$ & $\begin{array}{r}\text { Cases (VTE) } \\
\mathrm{n}=89\end{array}$ & $\begin{array}{r}\text { Controls (NORA) } \\
n=926\end{array}$ & p-Value \\
\hline Race & - & - & 0.018 \\
\hline Chinese & $42.7(38)^{a}$ & $50.8(470)$ & - \\
\hline Malay & $40.4(36)$ & $27.0(250)$ & - \\
\hline Indian & $12.4(11)$ & $10.8(100)$ & - \\
\hline Others & 4.49 (4) & $11.5(106)$ & - \\
\hline Age, years & $\begin{array}{r}31.6 \pm 6.13(18- \\
44)^{b}\end{array}$ & $\begin{array}{r}30.6 \pm 4.97(17- \\
45)\end{array}$ & 0.143 \\
\hline BMI, $\mathrm{kg} / \mathrm{m}^{2}$ & $\begin{array}{l}25.3 \pm 6.80 \\
(13.9-49.1)\end{array}$ & $\begin{array}{l}24.0 \pm 4.67 \\
(14.6-45.3)\end{array}$ & $0.234^{\circ}$ \\
\hline Parity & - & - & 0.012 \\
\hline 0 & $46.1(41)$ & $54.1(501)$ & - \\
\hline 1 & $29.2(26)$ & $31.9(295)$ & - \\
\hline 2 & $13.5(12)$ & $9.94(92)$ & - \\
\hline$\geq 3$ & $11.2(10)$ & $4.10(38)$ & - \\
\hline Non-O blood group & $81.9(68)$ & $58.0(536)$ & $<0.0001$ \\
\hline Smoking & $8.99(8)$ & $2.59(24)$ & 0.005 \\
\hline Pre-eclampsia & $5.80(4)$ & $2.16(20)$ & 0.078 \\
\hline Diabetes mellitus & $7.94(5)$ & $8.86(82)$ & 1 \\
\hline Caesarean delivery ${ }^{\mathrm{d}}$ & $55.2(16)$ & $32.1(297)$ & 0.014 \\
\hline $\begin{array}{l}\text { Gestational age at } \\
\text { delivery, weeks }\end{array}$ & $\begin{array}{l}35.9 \pm 4.72 \\
(24.3-40.3)\end{array}$ & $\begin{array}{l}38.7 \pm 1.53 \\
(25.0-41.7)\end{array}$ & $0.010^{\mathrm{e}}$ \\
\hline $\begin{array}{l}\text { Preterm delivery, } \\
<37 \text { weeks }^{d}\end{array}$ & $43.5(10)$ & $6.70(62)$ & $<0.0001$ \\
\hline $\begin{array}{l}\text { Newborn birth weight, } \\
\mathrm{kg}^{\mathrm{d}}\end{array}$ & $\begin{array}{l}2.61 \pm 0.93 \\
(0.69-4.02)\end{array}$ & $\begin{array}{l}3.11 \pm 0.46 \\
(0.80-4.50)\end{array}$ & $0.003^{\mathrm{e}}$ \\
\hline $\begin{array}{l}\text { Newborn LBW, } \\
<2.50 \mathrm{~kg}^{\mathrm{d}}\end{array}$ & $36.4(8)$ & $7.34(68)$ & 0.0002 \\
\hline $\begin{array}{l}\text { Postpartum haemor- } \\
\text { rhage, } \geq 500 \mathrm{~mL}^{\mathrm{d}}\end{array}$ & $27.3(6)$ & $11.8(64)$ & 0.043 \\
\hline
\end{tabular}

VTE, venous thromboembolism; BMI, body mass index; LBW, low birth weight; NORA, Neonatal and Obstetric Risk Assessment. ${ }^{a}$ Column \% (n). ${ }^{b}$ mean \pm standard deviation (minimum-maximum). ${ }^{c} p$-Value derived using $\ln \cdot \mathrm{BMI}$. ${ }^{\mathrm{d} V T E}$ cases that occurred in the postpartum period only $(n=29) .{ }^{e} p$-Values derived from Wilcoxon rank sum test.

obesity, rather than diabetes per se. While multiparity is an established component of the VTE risk assessment model [10], Malay race and non-O blood group are two identified factors which are inherent and easily identifiable during a woman's booking visit, and could potentially be added to the present VTE risk assessment model. Women with these characteristics should be followed up closely and regularly, with a heightened awareness for the development of VTE symptoms.

\section{Risk factors for postpartum VTE}

Caesarean section and preterm delivery were found to be significant risk factors for postpartum VTE. The finding of caesarean section as a risk factor for postpartum VTE is consistent with other studies [14] and can be attributed to endothelial wall damage from surgical intervention as well as venous stasis from postoperative immobilisation. This is clinically relevant in the Singapore obstetric population, in view of the rising rates of caesarean sections.

Although there were more VTE cases during the antepartum period, the number of VTE cases per week was considerably higher in the postpartum period, with the highest number of PE and DVT observed in the first postpartum week, steadily decreasing each week thereafter (Figure 2). In the postpartum period, a rise in the levels of coagulation factors occurs immediately after delivery [23], followed by the progressive return to pre-pregnancy concentrations over the six-week period [24, 25]. The findings in our study concurs with other studies [1, 2] underpinning the importance of postpartum VTE risk assessment and prophylaxis.

In this study, there were no cases of VTE diagnosed in the fifth or sixth week postpartum. Patients who delivered at these two hospitals would have been given a postpartum appointment (typically four weeks for caesarean delivery and six weeks for vaginal delivery). They would also be counselled to return earlier should they develop new symptoms, as they may be related to their pregnancy. Many of the VTE cases diagnosed after the first postpartum week were of patients that were discharged following delivery who returned to the same hospital. The scheduled postpartum follow-up allows the capture of postpartum VTE cases that may have been diagnosed outside of the two hospitals. However, due to our strict validation of cases (i.e. objective diagnosis), self-reported events would not have been included in our study.

\section{Strengths and limitations of the study}

All VTE cases were validated in this study. While ICD codes were used as the first tool to identify the VTE cases, all medical records were subsequently retrieved and reviewed, and only objectively-confirmed incident VTE cases were included in the study.

Like in most case-control studies, the selection of controls was a challenge. While the NORA cohort is assumed to be representative of the general obstetric population in Singapore, these women were studied from 2010 to 2014 in KKH, a subset of the period (2004-2016) and location (KKH and SGH) of this study. Intravenous fertilisation and ovarian hyperstimulation syndrome, which are known to be prothrombotic and could have contributed to a woman's VTE risk, or types of caesarean section (elective or emergency), could not be analysed in the logistic 
Table 2: Risk factors for pregnancy-associated VTE (logistic regression analysis).

\begin{tabular}{|c|c|c|c|c|c|c|}
\hline & \multicolumn{3}{|c|}{ Univariable analysis } & \multicolumn{3}{|c|}{ Multivariable analysis } \\
\hline & OR & $95 \% \mathrm{Cl}$ & p-Value & OR & $95 \% \mathrm{Cl}$ & p-Value \\
\hline Race, ref Chinese & - & - & 0.027 & - & - & 0.104 \\
\hline Malay & 1.78 & $1.10-2.87$ & 0.018 & 1.81 & $1.01-3.26$ & 0.048 \\
\hline Indian & 1.40 & $0.70-2.80$ & 0.345 & 1.30 & $0.54-3.13$ & 0.564 \\
\hline Others & 0.52 & $0.19-1.41$ & 0.197 & 0.59 & $0.20-1.75$ & 0.340 \\
\hline Age, ref $<30$ years & - & - & 0.108 & - & - & 0.198 \\
\hline 30-34 years old & 1.56 & $0.93-2.62$ & 0.089 & 1.78 & $0.95-3.34$ & 0.072 \\
\hline$\geq 35$ years old & 1.73 & $0.99-3.02$ & 0.053 & 1.48 & $0.71-3.05$ & 0.293 \\
\hline BMI, ref $18.5-24.9 \mathrm{~kg} / \mathrm{m}^{2}$ & - & - & 0.203 & - & - & - \\
\hline 25-29.9 (overweight) & 1.17 & $0.64-2.13$ & 0.604 & - & - & - \\
\hline$\geq 30$ (obese) & 1.85 & $0.94-3.62$ & 0.074 & - & - & - \\
\hline Parity, ref 0 & - & - & 0.013 & - & - & 0.147 \\
\hline 1 & 1.08 & $0.65-1.80$ & 0.757 & 1.11 & $0.60-2.04$ & 0.743 \\
\hline 2 & 1.63 & $0.83-3.20$ & 0.153 & 1.25 & $0.52-2.97$ & 0.622 \\
\hline$\geq 3$ & 3.30 & $1.54-7.04$ & 0.0002 & 2.84 & $1.16-6.97$ & 0.023 \\
\hline Non-O blood group, ref blood group 0 & 3.21 & $1.82-5.66$ & $<0.0001$ & 3.19 & $1.67-6.11$ & 0.0005 \\
\hline Smoker, ref non-smoker & 3.84 & $1.69-8.76$ & 0.001 & 5.44 & $2.21-14.00$ & 0.0002 \\
\hline Pre-eclampsia, ref no pre-eclampsia & 3.04 & $1.04-8.87$ & 0.042 & 2.76 & $0.87-8.77$ & 0.086 \\
\hline Diabetes, ref no diabetes & 0.96 & $0.39-2.39$ & 0.934 & - & - & - \\
\hline Factors specific to the postpartum period & - & - & - & - & - & - \\
\hline Caesarean section, ref vaginal delivery & 2.59 & $1.24-5.38$ & 0.011 & $3.58^{\mathrm{a}}$ & $1.07-12.02$ & 0.040 \\
\hline Preterm delivery ( $<37$ weeks), ref term delivery & 10.76 & $4.60-25.17$ & $<0.0001$ & $5.06^{\mathrm{a}}$ & $1.25-20.51$ & 0.023 \\
\hline Postpartum haemorrhage ( $\geq 500 \mathrm{~mL}$ ), ref no postpartum haemorrhage & 2.94 & $1.14-7.58$ & 0.026 & $1.71^{\mathrm{a}}$ & $0.44-6.62$ & 0.438 \\
\hline Newborn LBW (<2.50 kg), ref normal birth weight $(\geq 2.50 \mathrm{~kg})$ & 7.346 & $3.03-17.81$ & $<0.0001$ & $2.29^{\mathrm{a}}$ & $0.54-9.67$ & 0.260 \\
\hline
\end{tabular}

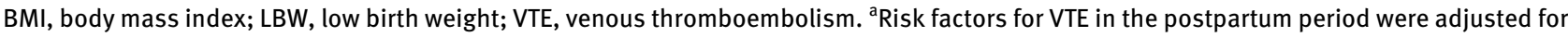
race, age, parity, blood group and presence of pre-eclampsia (overall p-value of $<0.2$ in the univariate analysis). None of these were found to be statistically significant risk factors for VTE in the postpartum period.

regression model as these data were unavailable in the NORA control group. Nonetheless, the advantage of using the NORA cohort as a control group was the availability of validated medical data and the ability to study potential risk factors like BMI and smoking - data not recorded in usual electronic medical record databases. We acknowledge the limitation of a smaller sample size affecting the statistical power of our analysis, producing wide confidence intervals around the estimated odds ratios. Ideally, VTE risk factors should be investigated in larger, multicentre cohorts.

\section{Conclusions}

Smoking, caesarean section and preterm delivery are conventional pregnancy-associated VTE risk factors identified in the study, as was multiparity. Notably, non-O blood group was also identified as a risk factor and Malay women were observed to be of greater risk in the Singapore obstetric population. The risk of developing
VTE extends throughout all three trimesters and beyond delivery to the six weeks postpartum. Screening using a VTE risk assessment tool to identify high-risk women who would benefit from thromboprophylaxis should therefore be conducted early in pregnancy and again just after delivery. This study offers a perspective on the burden of pregnancy-associated VTE in a Singaporean context, and should serve to inform policies regarding VTE thromboprophylaxis.

Research funding: None declared.

Author contributions: All authors have accepted responsibility for the entire content of this manuscript and approved its submission.

Competing interests: Authors state no conflict of interest. Informed consent: Informed consent was waivered in this study.

Ethical approval: The study was approved by the Ethics Committee SingHealth Centralised Institutional Review Board (CIRB) on 26 August 2016 (reference number 2016/ 2343). 


\section{References}

1. Heit JA, Kobbervig CE, James AH, Petterson TM, Bailey KR, Melton LJ. Trends in the incidence of venous thromboembolism during pregnancy or postpartum: a 30-year population-based study. Ann Intern Med 2005;143:697-706.

2. Sultan AA, West J, Tata LJ, Fleming KM, Nelson-Piercy C, Grainge MJ. Risk of first venous thromboembolism in and around pregnancy: a population-based cohort study. Br J Haematol 2012; 156:366-73.

3. Greer IA. Thrombosis in pregnancy: maternal and fetal issues. Lancet 1999;353:1258-65.

4. Meng K, Hu X, Peng X, Zhang Z. Incidence of venous thromboembolism during pregnancy and the puerperium: a systematic review and meta-analysis. J Matern Neonatal Med 2015;28:245-53.

5. Kourlaba G, Relakis J, Kontodimas S, Holm MV, Maniadakis N. A systematic review and meta-analysis of the epidemiology and burden of venous thromboembolism among pregnant women. Int J Gynecol Obstet 2016;132:4-10.

6. Say L, Chou D, Gemmill A, Tuncalp O, Moller A, Daniels J, et al. Global causes of maternal death: a WHO systematic analysis. Lancet Glob Heal 2014;2:323-33.

7. Lau G. Are maternal deaths on the ascent in Singapore? A review of maternal mortality as reflected by coronial casework from 1990 to 1999. Ann Acad Med Singapore 2002;31:261-75.

8. Wik HS, Jacobsen AF, Sandvik L, Sandset PM. Prevalence and predictors for post-thrombotic syndrome 3-16 years after pregnancy-related venous thrombosis: a population-based, cross-sectional, case-control study. J Thromb Haemostasis 2012; 10:840-7.

9. Wik HS, Enden TR, Jacobsen AF, Sandset PM. Long-term quality of life after pregnancy-related deep vein thrombosis and the influence of socioeconomic factors and comorbidity. J Thromb Haemostasis 2011;9:1931-6.

10. RCOG. Reducing the risk of venous thromboembolism during pregnancy and the puerperium: Green-Top Guideline No. 37a; 2015.

11. Liew NC, Alemany GV, Angchaisuksiri P, Bang SM, Choi G, De Silva DA, et al. Asian venous thromboembolism guidelines: updated recommendations for the prevention of venous thromboembolism. Int Angiol 2017;36:1-20.

12. Won HS, Kim DY, Yang MS, Lee SJ, Shin HH, Parl JB. Pregnancyinduced hypertension, but not gestational diabetes mellitus, is a risk factor for venous thromboembolism in pregnancy. Korean Circ J 2011;41:23-7.

13. Gader AA, Haggaz AED, Adam I. Epidemiology of deep venous thrombosis during pregnancy and puerperium. Vasc Health Risk Manag 2009;5:2007-10.

14. Kevane B, Donnelly J, D’Alton M, Cooley S, Preston RJS, Ainle FN. Risk factors for pregnancy-associated venous thromboembolism: a review. J Perinat Med 2014;42:417-25.

15. Larsen TB, Johnsen SP, Gislum M, Møller CAl, Larsen H, Søresnsen HT. ABO blood groups and risk of venous thromboembolism during pregnancy and the puerperium. A population-based, nested case-control study. J Thromb Haemostasis 2005;3:300-4.

16. Blondon M, Quon BS, Harrington LB, Bounameaux H, Smith NL. Association between newborn birth weight and the risk of postpartum maternal venous thromboembolism a populationbased case-control study. Circulation 2015;131:1471-6.

17. Lim WY, Saw SM, Tan KH, Yeo GSH, Kwek KYC. A cohort evaluation on arterial stiffness and hypertensive disorders in pregnancy. BMC Pregnancy Childbirth 2012;12:160.

18. Rasmussen KM, Catalano PM, Yaktine AL. New guidelines for weight gain during pregnancy: what obstetrician/gynecologists should know. Curr Opin Obstet Gynecol 2009;21:521-6.

19. Molina JAD, Gabriel ZJ, Heng BH. Venous thromboembolism at the National Healthcare Group, Singapore. Ann Acad Med Singapore 2009;38:470-7.

20. White RH, Keenan CR. Effects of race and ethnicity on the incidence of venous thromboembolism. Thromb Res 2009;123: S11-7.

21. Robert A, Aillaud MF, Eschwège V, Randrianjohany A, Scarabin $Y$, Juhan-Vague I. ABO blood group and risk of venous thrombosis in heterozygous carriers of factor V Leiden. Thromb Haemostasis 2000;83:630-1.

22. Heit JA, Leibson CL, Ashrani AA, Petterson TM, Bailey KR, Melton LJ. Is diabetes mellitus an independent risk factor for venous thromboembolism? A population-based case-control study. Arterioscler Thromb Vasc Biol 2009;29:1399-405.

23. Bonnar J, McNicol GP, Douglas AS. Coagulation and fibrinolytic mechanisms during and after normal childbirth. Br Med J 1970;2: 200-3.

24. Boehlen F, Epiney M, Boulvain M, Reber G, Antonelli E, Morales M, et al. D-dimer levels during the pregnancy and the postpartum. Rev Med Suisse 2005;1:296-8.

25. Kjellberg U, Andersson NE, Rosén S, Tengborn L, Hellgren M. APC resistance and other haemostatic variables during pregnancy and puerperium. Thromb Haemostasis 1999;81:527-31. 